\title{
A CHARACTERISATION OF NEWTON MAPS
}

\author{
A. BERGER ${ }^{\varpi 1}$ and T. P. HILL ${ }^{2}$
}

(Received 10 December, 2005)

\begin{abstract}
Conditions are given for a $C^{k}$ map $T$ to be a Newton map, that is, the map associated with a differentiable real-valued function via Newton's method. For finitely differentiable maps and functions, these conditions are only necessary, but in the smooth case, that is, for $k=\infty$, they are also sufficient. The characterisation rests upon the structure of the fixed point set of $T$ and the value of the derivative $T^{\prime}$ there, and it is best possible as is demonstrated through examples.
\end{abstract}

2000 Mathematics subject classification: primary 37N30, 39A11, 49M15; secondary 37E05, $65 \mathrm{H} 05$.

Keywords and phrases: Newton's method, Newton map, fixed point set, attracting fixed point.

\section{Introduction}

Newton's method (NM) for computing successive approximations of zeros of functions is one of the most widely used methods in all of applied mathematics; variants and generalisations also play a prominent role in numerous other disciplines $[2,3,6,8,9]$. Conceptually, NM becomes especially transparent within a dynamical systems context. The purpose of this brief note is to characterise, in the simplest possible setting, the local properties of the dynamical systems thus encountered.

Throughout, let $f: I \rightarrow \mathbb{R}$ be a differentiable function, defined on some open interval $I \subset \mathbb{R}$, and denote by $N_{f}$ its associated NM transformation, that is,

$$
N_{f}(x)=x-\frac{f(x)}{f^{\prime}(x)}, \quad \text { for all } x \in I: f^{\prime}(x) \neq 0
$$

\footnotetext{
${ }^{1}$ Department of Mathematics and Statistics, University of Canterbury, Christchurch, New Zealand; e-mail: arno.berger@canterbury.ac.nz.

${ }^{2}$ School of Mathematics, Georgia Institute of Technology, Atlanta, USA; e-mail: hill@math.gatech.edu. (C) Australian Mathematical Society 2006, Serial-fee code 1446-1811/06
} 
for $N_{f}$ to be defined for every $x \in I$, set $N_{f}(x):=x$ whenever $f^{\prime}(x)=0$.

NM for finding roots (zeros) of $f$, that is, real numbers $x^{*}$ with $f\left(x^{*}\right)=0$, amounts to picking an initial point $x_{0} \in I$ and iterating $N_{f}$, thus generating the sequence

$$
x_{n}=N_{f}\left(x_{n-1}\right)=N_{f}^{n}\left(x_{0}\right), \quad \text { for all } n \in \mathbb{N},
$$

where, here and throughout, for any map $T: I \rightarrow \mathbb{R}$ and any $n \in \mathbb{N}, T^{n}(x)=$ $T\left(T^{n-1}(x)\right)$, provided that $T^{n-1}(x) \in I$, and $T^{0}(x)=x$. Note that $N_{f}(x)=x$ precisely if $f(x) f^{\prime}(x)=0$; that is, the only fixed points of $N_{f}$ occur where either $f$ or $f^{\prime}$ vanish. Thus for $f\left(x_{n}\right) f^{\prime}\left(x_{n}\right)=0$, and only then, does NM terminate at $x_{n}$. If $f\left(x_{n}\right)=0$, a root has been found, and otherwise (1.1) breaks down due to a horizontal tangent to the graph of $f$ at $x_{n}$ (see Figure 1).

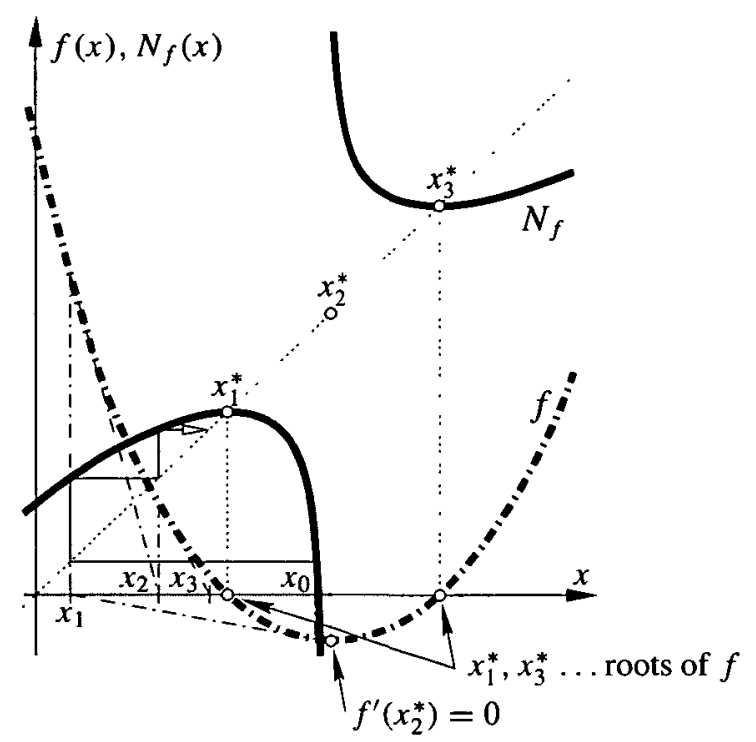

FIGURE 1. Visualising NM: The first few iterates $x_{1}, x_{2}, x_{3}$ are found graphically, both by means of tangents to the graph of $f$ (broken line) and via the graph of $N_{f}$ (solid line). Note how the point $x_{2}^{*}$ with $f^{\prime}\left(x_{2}^{*}\right)=0$ causes $N_{f}$ to have a discontinuity.

Clearly, if $\left(x_{n}\right)$ converges to $x^{*}$, say, and if $N_{f}$ is continuous at $x^{*}$, then $N_{f}\left(x^{*}\right)=x^{*}$, that is, $x^{*}$ is a fixed point of $N_{f}$, and $f\left(x^{*}\right)=0$. (The trivial alternative $f \equiv$ const. is tacitly excluded here, see Lemma 2.4 below.) It is this correspondence between the roots of $f$ and the fixed points of $N_{f}$ that suggests that NM be studied as a dynamical system. Under a mild assumption, each (isolated) fixed point $x^{*}$ is attracting, that is, $\lim _{n \rightarrow \infty} N_{f}^{n}\left(x_{0}\right)=x^{*}$ for all $x_{0}$ sufficiently close to $x^{*}$. (For $x_{0}$ further away from any root, the sequence $\left(x_{n}\right)$ may exhibit a considerably more complicated long-term behaviour $[2,3,9]$.) This aspect of NM is put into perspective by the main result 
of the present paper, Theorem 3.2 below, which completely characterises the local dynamical properties of $N_{f}$.

\section{Newton maps}

The definition of a Newton map given below entails a relationship between the analytic properties of a function $f$ and the analytic properties of its associated NM transformation $N_{f}$. It is a simple fact, rarely alluded to in studies of NM, that in general these properties are quite independent.

EXAMPLE 2.1. The function $f(x)=|x|^{3 / 2}$ is $C^{1}$ but not $C^{2}$, yet it has a $C^{\infty} \mathrm{NM}$ transformation, namely $N_{f}(x)=x / 3$.

EXAMPLE 2.2. It is easily seen that the function

$$
f(x)= \begin{cases}\exp \left(-x^{-2}+|x|+\cos \left(x^{-2}\right)\right) & \text { if } x \neq 0 \\ 0 & \text { if } x=0\end{cases}
$$

is $C^{\infty}$, and both $f$ and $f^{\prime}$ vanish only at $x^{*}=0$. Nevertheless

$$
-1=\liminf _{x \rightarrow 0} N_{f}(x)<\limsup _{x \rightarrow 0} N_{f}(x)=1,
$$

hence $N_{f}$ is not even continuous at $x^{*}$.

Since $N_{f}$ may fail to be continuous even if $f$ is $C^{\infty}$, in order to ensure the applicability of NM, some explicit assumption on the smoothness of $N_{f}$ has to be imposed. To formulate such conditions concisely, let $\mathbb{N}_{\infty}=\mathbb{N} \cup\{\infty\}$ and stipulate that $\infty^{-1}:=0$ and $\infty \pm j=\infty$ for all $j \in \mathbb{N}$.

In view of (1.1), for $N_{f}$ to be $C^{l}$ for some $l \in \mathbb{N}_{\infty}$, one might demand that $f$ be at least $C^{l+1}$, but Examples 2.1 and 2.2 show that this assumption is neither necessary nor sufficient. Simply imposing further conditions on $N_{f}$ also seems problematic as long as it is not clear whether any such condition is satisfied for a reasonably large class of functions. Thus it is inevitable to address the following general inverse problem: Given a $C^{l}$ map $T$, does there exist a function $f$ such that $T=N_{f}$ ?

DEFINITION 2.3. Let $I \subset \mathbb{R}$ be an open interval, and $l \in \mathbb{N}_{\infty}$. A map $T \in C^{l}(I)$ is called a Newton map (associated with $f$ ), if $T=N_{f}$ for some differentiable function $f: I \rightarrow \mathbb{R}$.

Clearly, not every $T \in C^{l}(I)$ is a Newton map, even if $l=\infty$, as the trivial example $T(x)=-x$ shows, for which every $f$ with $N_{f}=T$ lacks differentiability at 
$x^{*}=0$. As will become clear shortly, most maps are not Newton, but a satisfactory characterisation is not available for finitely differentiable maps. However, in the smooth case, that is, for $l=\infty$, there is a simple characterisation of Newton maps, as provided by Theorem 3.2 below.

For any map $T$, denote by Fix $[T]$ the set of fixed points of $T$, that is, $\operatorname{Fix}[T]:=$ $\{x \in I: T(x)=x\}$, and say that Fix $[T]$ is attracting if $\lim _{n \rightarrow \infty} T^{n}\left(x_{0}\right) \in \operatorname{Fix}[T]$ for all $x_{0}$ sufficiently close to Fix $[T]$.

LEMMA 2.4. Let $f: I \rightarrow \mathbb{B}$ be differentiable, and assume that $N_{f}$ is continuous. Then Fix $\left[N_{f}\right]$ is either empty or a (possibly one-point) interval; in the latter case,

$$
\limsup _{x \rightarrow x^{*}} \frac{N_{f}(x)-x^{*}}{x-x^{*}}=\delta \quad \text { for some } \delta \in[0,1]
$$

holds for every $x^{*} \in \operatorname{Fix}\left[N_{f}\right]$.

PROOF. It will first be shown that both sets $Z_{0}:=\{x \in I: f(x)=0\}$ and $Z_{1}:=\left\{x \in I: f^{\prime}(x)=0\right\}$ of zeros of $f$ and $f^{\prime}$, respectively, are (possibly empty or one-point) subintervals of $I$. Moreover, if $Z_{1} \neq I$, that is, if $f$. is not constant, then $Z_{1} \subset Z_{0}$; in fact, the two sets coincide unless $Z_{0}$ contains exactly one point, in which case $Z_{1}$ may be empty. Since $\operatorname{Fix}\left[N_{f}\right]=Z_{0} \cup Z_{1}$ the first part of the lemma follows immediately from this.

If $Z_{1}=I$, then Fix $\left[N_{f}\right]=I$, so let $Z_{1} \neq \emptyset$ be different from $I$. Pick $a \in Z_{1}$, suppose, by way of contradiction, $f(a) \neq 0$ and, without loss of generality, that $b:=\sup \{x \geq a: f(y)=f(a)$ for all $y \in[a, x]\}$ belongs to $I$. Clearly, $f(b)=f(a)$ and $f^{\prime}(b)=0$, hence $N_{f}(b)=b$. By the Mean Value Theorem there exists a sequence $b_{n} \searrow b$ such that $0<\left|f^{\prime}\left(b_{n}\right)\right| \leq 1$ for all $n$. But then

$$
\liminf _{n \rightarrow \infty}\left|N_{f}\left(b_{n}\right)-b\right| \geq \lim _{n \rightarrow \infty}\left|f\left(b_{n}\right)\right|=|f(b)|=|f(a)|>0,
$$

clearly contradicting the continuity of $N_{f}$. Therefore $f(a)=0$, hence $Z_{1} \subset Z_{0}$. If $a_{1}<a_{2}$ both belong to $Z_{0}$ then, by the previous argument and the Mean Value Theorem, $Z_{0}$ contains a point strictly between $a_{1}$ and $a_{2}$. Since $Z_{0}$ is closed, it contains, with any two points, the whole segment joining these points. Thus $Z_{0}$ is an interval. If $Z_{0}$ is not a singleton then $Z_{0} \subset Z_{1}$ and therefore $Z_{0}=Z_{1}$. The latter equality also holds if $Z_{0}$ is one-point because $Z_{1} \neq \emptyset$. Finally, if $Z_{1}$ is empty then clearly $Z_{0}$ cannot contain more than one point.

Assertion (2.1) is trivially true if $x^{*}$ is an interior point of Fix $\left[N_{f}\right]$. Without loss of generality therefore assume that $x^{*}$ is, say, a right boundary point of Fix $\left[N_{f}\right]=Z_{0}$. Choose $\delta>0$ so small that $\left.J:=] x^{*}, x^{*}+\delta\right] \subset I$ and, for $0<t \leq \delta$, let

$$
h(t):=\frac{N_{f}\left(x^{*}+t\right)-x^{*}}{t}
$$


the function $h$ is continuous on $] 0, \delta]$, and $h(t) \neq 1$ for all $t>0$. Since $x \neq N_{f}(x)$ for $x \in J$,

$$
\frac{f^{\prime}(x)}{f(x)}=\frac{1}{x-N_{f}(x)}, \quad \text { for all } x \in J
$$

which after integrating both sides from $x$ to $x^{*}+\delta$, and using the auxiliary function $h$ defined in (2.2), can be written as

$$
f(x)=f\left(x^{*}+\delta\right) \exp \left(-\int_{x-x^{*}}^{\delta} \frac{1}{1-h(t)} \frac{d t}{t}\right), \quad \text { for all } x \in J
$$

Assume $f\left(x^{*}+\delta\right)>0$ without loss of generality. If $h(t)>1$ for all $t>0$, then (2.3) implies that $f\left(x^{*}\right) \neq 0$, contradicting $x^{*} \in Z_{0}$. Thus $h(t)<1$ for all $t>0$, and in particular

$$
\limsup _{t \searrow 0} h(t)=\limsup _{x \searrow x^{*}} \frac{N_{f}(x)-x^{*}}{x-x^{*}} \leq 1
$$

Fix $j \in \mathbb{N}$. Dividing (2.3) by $\left(x-x^{*}\right)^{j}=\delta^{j} \exp \left(-j \int_{x-x^{*}}^{\delta} d t / t\right)$ yields

$$
\frac{f(x)}{\left(x-x^{*}\right)^{\prime}}=\frac{f\left(x^{*}+\delta\right)}{\delta^{J}} \exp \left(\int_{x-x^{*}}^{\delta} \frac{j-1-j h(t)}{1-h(t)} \frac{d t}{t}\right), \quad \text { for all } x \in J
$$

To bound lim $\sup _{t \backslash 0} h(t)$ from below, pick $\varepsilon>0$ and assume that $h(t)<-\varepsilon$ for all sufficiently small $t>0$. In this case, (2.4) with $j=1$ shows that

$$
\left(x-x^{*}\right)^{-1} f(x) \geq f\left(x^{*}+\delta\right) \delta^{-(1+\varepsilon)^{-1}}\left(x-x^{*}\right)^{-\varepsilon(1+\varepsilon)^{-1}} \rightarrow \infty, \quad \text { as } x \searrow x^{*},
$$

which contradicts the differentiability of $f$ at $x^{*}$. Since $\varepsilon>0$ was arbitrary, $\lim \sup _{t \searrow 0} h(t) \geq 0$.

REMARK. (i) Lemma 2.4 should be contrasted with the simple fact that for every closed set $A \subset \mathbb{R}$ there exists a $C^{\infty}$ map $T$ with $T(I) \subset I$ and $\operatorname{Fix}[T]=A \cap I$.

(ii) Under the conditions of Lemma 2.4 there is no analogue to (2.1) for the corresponding lim inf which, as simple examples show, can be any number between, and including, the trivial bounds $-\infty$ and $\delta$.

As pointed out earlier, the applicability of NM rests on the correspondence between the roots of $f$ and the fixed points of $N_{f}$ - and the attractiveness of the latter. Mere continuity of $N_{f}$ does not guarantee that $\operatorname{Fix}\left[N_{f}\right]$ is attracting. 
EXAMPLE 2.5. Consider the $C^{1}$ function

$$
f(x)= \begin{cases}|x|^{3 / 2} \exp \left(-\int_{0}^{|x|^{-1}} t^{-1} \sin t d t\right) & \text { if } x \neq 0 \\ 0 & \text { if } x=0\end{cases}
$$

for which the associated NM transformation

$$
N_{f}(x)= \begin{cases}x\left(1+2 \sin \left(|x|^{-1}\right)\right) /\left(3+2 \sin \left(|x|^{-1}\right)\right) & \text { if } x \neq 0 \\ 0 & \text { if } x=0\end{cases}
$$

is continuous yet obviously not $C^{1}$. The only fixed point of $N_{f}$, and correspondingly the only root of $f$ and $f^{\prime}$, is $x^{*}=0$. Since, for every $j \in \mathbb{N}$, the points $\pm 2 /(\pi(4 j-1))$ are 2-periodic, $\operatorname{Fix}\left[N_{f}\right]=\{0\}$ is not attracting.

Thus while $\operatorname{Fix}\left[N_{f}\right]$ is topologically simple whenever $N_{f}$ is continuous, to make NM practical for approximating zeros, more smoothness is required. Only the case of $N_{f}$ being at least $C^{1}$ will therefore be considered from now on. (For the same reason, the legitimate case $l=0$ has been excluded from Definition 2.3.) Also, the properties of $N_{f}^{\prime}$, albeit not completely determined by the smoothness of $f$, do depend on the latter. To describe this dependence, for every $k \in \mathbb{N}_{\infty}$, define the set

$$
\left.\left.\Delta_{k}:=\left\{0,1 / 2,2 / 3, \ldots, 1-k^{-1}\right\} \cup\right] 1-k^{-1}, 1\right]
$$

and note that $[0,1]=\Delta_{1} \supset \Delta_{2} \supset \cdots \supset \Delta_{\infty}=\left\{1-j^{-1}: j \in \mathbb{N}_{\infty}\right\}$.

LEMMA 2.6. Let $f: I \rightarrow \mathbb{R}$ be differentiable, and assume that $N_{f} \in C^{1}(I)$. Then $\operatorname{Fix}\left[N_{f}\right]$ is either empty or an attracting (possibly one-point) interval. Moreover, if Fix $\left[N_{f}\right] \neq \emptyset$ and $f \in C^{k}(I)$ with $k \in \mathbb{N}_{\infty}$ then

$$
N_{f}^{\prime}\left(\operatorname{Fix}\left[N_{f}\right]\right)=\{\delta\} \quad \text { for some } \delta \in \Delta_{k} .
$$

ProOF. The assertions are trivially true if $f$ is constant or Fix $\left[N_{f}\right]=\emptyset$. Therefore assume that $f$ is not constant and Fix $\left[N_{f}\right]$ is not empty, hence a subinterval of $I$, by Lemma 2.4. If $x^{*}$ is an interior point of Fix $\left[N_{f}\right]$ then $N_{f}^{\prime} \equiv 1$ in a neighbourhood of $x^{*}$, and the assertion is again true. Thus assume without loss of generality that $x^{*}$ is a right boundary point of Fix $\left[N_{f}\right]$. By Lemma $2.4, N_{f}^{\prime}\left(x^{*}\right) \in \Delta_{1}$, so $x^{*}$ obviously is attracting from the right, unless perhaps for $N_{f}^{\prime}\left(x^{*}\right)=1$. In the latter case, with the notation introduced in the proof of Lemma 2.4, the function $h$ defined in (2.2), supplemented by $h(0):=N_{f}^{\prime}\left(x^{*}\right)=1$, is continuous on $[0, \delta]$ and can be written as $h(t)=1-H(t)$, where $H$ is also continuous on $[0, \delta]$, and $H(t) \neq 0$ unless $t=0$. With this, (2.3) takes the form

$$
f(x)=f\left(x^{*}+\delta\right) \exp \left(-\int_{x-x^{*}}^{\delta} \frac{d t}{t H(t)}\right), \quad \text { for all } x \in J .
$$


Since $f\left(x^{*}\right)=0$ and $f\left(x^{*}+\delta\right) \neq 0$, the integral $\int_{0}^{\delta} d t /(t H(t))$ must diverge to $+\infty$. As $H$ is continuous and, except possibly at $t=0$, does not change sign, $H(t)>0$ and so $h(t)<1$ whenever $0<t \leq \delta$. From $N_{f}\left(x^{*}+t\right)-x^{*}=t h(t)<t$ and $h(0)=0$ it follows that $x^{*}<N_{f}\left(x_{0}\right)<x_{0}$ and therefore $N_{f}^{n}\left(x_{0}\right) \searrow x^{*}$ provided that $x_{0} \in J$. In other words, $x^{*}$ is attracting from the right.

It remains to verify (2.6) for $f \in C^{k}(I)$. To this end, assume first that $k<\infty$ and $f\left(x^{*}\right)=f^{\prime}\left(x^{*}\right)=\cdots=f^{(k)}\left(x^{*}\right)=0$. In this case, since $f$ is $C^{k}$, the left-hand side in (2.4) with $j=k$ tends to a finite limit as $x \searrow x^{*}$. Consequently,

$$
\lim _{\varepsilon \searrow 0} \int_{\varepsilon}^{\delta} \frac{k-1-k h(t)}{1-h(t)} \frac{d t}{t}<+\infty .
$$

If $h(0)<1-k^{-1}$, then the integrand in (2.7) would eventually be positive near $t=0$, which clearly is impossible. Therefore $h(0) \geq 1-k^{-1}$. Since $h(0) \leq 1$ by the same argument,

$$
N_{f}^{\prime}\left(x^{*}\right)=h(0) \in\left[1-k^{-1}, 1\right] \subset \Delta_{k} .
$$

If $k=\infty$ and $f^{(J)}\left(x^{*}\right)=0$ for all $j \in \mathbb{N}$, then similar reasoning shows that $N_{f}^{\prime}\left(x^{*}\right) \in \bigcap_{j \in \mathbb{N}}\left[1-j^{-1}, 1\right]=\{1\} \subset \Delta_{\infty}$.

Finally assume that $f\left(x^{*}\right)=f^{\prime}\left(x^{*}\right)=\cdots=f^{(j)}\left(x^{*}\right)=0$ yet $f^{(j+1)}\left(x^{*}\right) \neq 0$ for some $j$ with $0 \leq j<k$. The same argument as before with $k$ replaced by $j$ shows that $N_{f}^{\prime}\left(x^{*}\right) \in\left[1-(j+1)^{-1}, 1\right]$. If $h(0)>1-(j+1)^{-1}$, then (2.4) with $j$ replaced by $j+1$ would imply that $\lim _{x \backslash x^{*}}\left(x-x^{*}\right)^{-(j+1)} f(x)=0$, which contradicts $f^{(j+1)}\left(x^{*}\right) \neq 0$. Thus $N_{f}^{\prime}\left(x^{*}\right)=h(0)=1-(j+1)^{-1} \in \Delta_{\infty} \subset \Delta_{k}$.

EXAMPLE 2.7. Lemma 2.6 is best possible in the following sense: For every $k \in \mathbb{N}_{\infty}$ and $\delta \in \Delta_{k}$ there exists a $C^{k}$ function $f$ with $N_{f} \in C^{1}$ having a single fixed point $x^{*}$ such that $N_{f}^{\prime}\left(x^{*}\right)=\delta$. For $k \in \mathbb{N}$ and $\delta \in \Delta_{k} \backslash\{1\}$ let $\gamma=(1-\delta)^{-1}$ and consider the function

$$
f(x)= \begin{cases}x^{\gamma}\left(1+(2 k+4)^{-1} x^{(1+\gamma)(1+k)} \sin \left(x^{-\gamma}\right)\right) & \text { if } 0<|x|<1, \\ 0 & \text { if } x=0,\end{cases}
$$

where, for non-integer $\gamma$, each argument $x$ has to be replaced by $|x|$. Taking $I=$ ] $-1,1$ [, it is readily checked that $f \in C^{k}(I)$ and $N_{f} \in C^{1}(I)$. Moreover, $x^{*}=0$ is the only fixed point of $N_{f}$ in $I$, and $N_{f}^{\prime}\left(x^{*}\right)=1-\gamma^{-1}=\delta$. For $\delta=1$, an example is provided by the $C^{k}$ function

$$
f(x)=e^{-1 /|x|}+\frac{1}{2} e^{-(k+4) /|x|} \sin \left(e^{1 /|x|}\right),
$$

for which $N_{f}$ is $C^{1}$, has $x^{*}=0$ as its only fixed point, and $N_{f}^{\prime}\left(x^{*}\right)=1$. Simple examples in the case $k=\infty$ are $f(x)=x^{\gamma}$ for $\delta<1$, and $f(x)=\exp \left(-|x|^{-1}\right)$ for $\delta=1$, respectively. 
An important special case for which Lemma 2.6 can be strengthened is the case of a root of finite multiplicity. Recall that $x^{*} \in I$ is a root of $f \in C^{k}(I)$ of multiplicity $j \in \mathbb{N}$ if $f(x)=\left(x-x^{*}\right)^{j} g(x)$ for all $x \in I$, where $g \in C^{k}(I)$ and $g\left(x^{*}\right) \neq 0$.

LEMMA 2.8. Let $x^{*}$ be a root of $f \in C^{k}(I)$ of finite multiplicity $j$. Then, for some open interval $J \subset I$ containing $x^{*}, N_{f} \in C^{k-1}(J)$, and $N_{f}^{\prime}\left(x^{*}\right)=1-j^{-1}$; in particular, $\operatorname{Fix}\left[N_{f}\right] \cap J=\left\{x^{*}\right\}$ is attracting.

ProOF. Since $f(x)=\left(x-x^{*}\right)^{j} g(x)$ for some $g \in C^{k}$ with $g\left(x^{*}\right) \neq 0$,

$$
N_{f}(x)-x^{*}=\left(x-x^{*}\right) \frac{(j-1) g(x)+\left(x-x^{*}\right) g^{\prime}(x)}{j g(x)+\left(x-x^{*}\right) g^{\prime}(x)}=\left(x-x^{*}\right) h(x),
$$

where $h$ is $C^{k-1}$ on some open interval $J \subset I$ containing $x^{*}$, and

$$
N_{f}^{\prime}\left(x^{*}\right)=h\left(x^{*}\right)=1-j^{-1} \text {. }
$$

Thus, for $J$ chosen sufficiently small, Fix $\left[N_{f}\right] \cap J=\left\{x^{*}\right\}$, and the fixed point $x^{*}$ clearly is attracting.

\section{Main theorem}

Lemma 2.6 contains necessary conditions for a map to be Newton. In general it is too much to expect that every $T \in C^{1}(I)$ whose fixed point set is attracting and satisfies (2.6) would be a Newton map associated with some $f \in C^{k}(I)$.

EXAMPLE 3.1. Let $I=]-1,1[$ and consider the map

$$
T(x)= \begin{cases}x / \log |x| & \text { if } 0<|x|<1, \\ 0 & \text { if } x=0,\end{cases}
$$

which has $x^{*}=0$ as its only and attracting fixed point and, with $T^{\prime}\left(x^{*}\right):=0$, is $C^{1}$ on $I$. Obviously $T^{\prime}\left(x^{*}\right) \in \Delta_{k}$ for all $k \in \mathbb{N}_{\infty}$. Suppose that $N_{f}=T$ for some $f \in C^{k}(I)$. Then, with some nonzero constant $C$,

$$
f(x)=C x(1-\log x), \text { for all } x: 0<x<1 .
$$

Clearly, this function cannot be extended to even a differentiable function on $I$. Thus $N_{f} \neq T$ for every $f \in C^{k}(I)$. The fact that in this example $T$ is barely $C^{1}$ is not important, as it is easy to find similar examples with $T$ showing any finite degree of differentiability: For every $l \in \mathbb{N}$ (and $k \in \mathbb{N}_{\infty}$ ) there exist maps $T \in C^{\prime}(I)$ such that $T^{\prime}($ Fix $[T])=\{\delta\}$ with $\delta \in \Delta_{k}$, yet $N_{f} \neq T$ for all $f \in C^{k}(I)$. 
Example 3.1 shows that there is no hope for a converse of Lemma 2.6 to hold, even if $N_{f}$ is assumed to be more regular than $C^{1}$. However, the situation is much clearer for smooth maps, that is, for $l=\infty$. In this case, the converse of Lemma 2.6 does actually hold, that is, the stated conditions are also sufficient.

THEOREM 3.2. Let $k \in \mathbb{N}_{\infty}$, and suppose $T \in C^{\infty}(I)$. Then $T$ is a Newton map, associated with $f \in C^{k}(I)$, if and only if $\operatorname{Fix}[T]$ either is empty or an attracting (possibly one-point) interval, and

$$
T^{\prime}(\operatorname{Fix}[T])=\{\delta\}, \quad \text { for some } \delta \in \Delta_{k} .
$$

Moreover, the function $f$ is uniquely determined up to a multiplicative constant if either $\delta \in\left\{0,1 / 2,1 / 3, \ldots, 1-k^{-1}\right] \backslash\{1\}$ or the set $I \backslash \mathrm{Fix}[T]$ is connected.

ProOF. If $T$ is a Newton map then, by Lemma 2.6, Fix $[T]$ is an attracting interval (which may be empty or one-point), and (3.1) holds. Thus only the converse statement and the uniqueness assertion have yet to be proved. To this end, three cases will be distinguished; throughout let $g(x):=x-T(x)$.

Case 1. Assume that Fix $[T]=\emptyset$. Then $g$ is nonvanishing and $C^{\infty}$ on $I$, and so is

$$
f(x)=\exp \left(\int_{\xi}^{x} \frac{d t}{g(t)}\right), \quad \text { for all } x \in I,
$$

where $\xi$ is any point in $I$. Since $g$ is $C^{\infty}$ and does not vanish on $I$, the solution $f$ of the first-order ODE $f^{\prime} / f=1 / g$, or equivalently, $N_{f}=T$, is unique up to multiplication by a constant.

Case 2. Assume that $x^{*} \in \operatorname{Fix}[T]$ and $T^{\prime}\left(x^{*}\right)=\delta$ with $\delta \in \Delta_{k} \backslash\{1\}$. Clearly this implies that Fix $[T]=\left\{x^{*}\right\}$, and $T$ can be written as

$$
T(x)=x^{*}+\delta\left(x-x^{*}\right)+(1-\delta)\left(x-x^{*}\right)^{2} h(x),
$$

with a uniquely determined $h \in C^{\infty}$. Note that $\left(x-x^{*}\right) h(x) \neq 1$ for all $x \in I$. Let $\gamma=(1-\delta)^{-1}$, pick points $x^{-}, x^{+} \in I$ with $x^{-}<x^{*}<x^{+}$, and define $f: I \rightarrow \mathbb{R}$ by

$$
f(x):= \begin{cases}c^{+}\left(x^{+}-x^{*}\right)^{\gamma} \exp \left(-\int_{x}^{x^{+}} d t / g(t)\right) & \text { if } x>x^{*}, \\ 0 & \text { if } x=x^{*}, \\ c^{-}\left(x^{*}-x^{-}\right)^{\gamma} \exp \left(\int_{x^{-}}^{x} d t / g(t)\right) & \text { if } x<x^{*} ;\end{cases}
$$

here $c^{+}, c^{-}$are nonzero real constants. Since $x^{*}$ is the only fixed point of $T$ in $I$ it follows that $f \in C^{\infty}\left(I \backslash\left\{x^{*}\right\}\right)$, and $N_{f}=T$. By using the identity

$$
\left(x-x^{*}\right)^{\gamma}=\left(x^{+}-x^{*}\right)^{\gamma} \exp \left(-\gamma \int_{x}^{x^{+}} \frac{d t}{t-x^{*}}\right), \text { for all } x>x^{*}
$$


a short computation yields

$$
\left(x-x^{*}\right)^{-\gamma} f(x)=c^{+} \exp \left(-\gamma \int_{x}^{x^{+}} \frac{h(t) d t}{1-\left(t-x^{*}\right) h(t)}\right), \quad \text { for all } x>x^{*} .
$$

An analogous computation for $x<x^{*}$ yields

$$
\left(x^{*}-x\right)^{-\gamma} f(x)=c^{-} \exp \left(\gamma \int_{x^{-}}^{x} \frac{h(t) d t}{1-\left(t-x^{*}\right) h(t)}\right), \quad \text { for all } x<x^{*} .
$$

Since the integrand $h(t) /\left(1-\left(t-x^{*}\right) h(t)\right)$ is $C^{\infty}$ on $I$, both one-sided limits for $\left|x-x^{*}\right|^{-\gamma} f(x)$, as $x$ approaches $x^{*}$, are finite and nonzero. If $\delta=1-j^{-1}$ for some $1 \leq j \leq k$ then, for $f$ to be $C^{\jmath}$ on $I$, these two one-sided limits have to be equal or, equivalently,

$$
c^{-}=(-1)^{j} c^{+} \exp \left(-j \int_{x^{-}}^{x^{+}} \frac{h(t) d t}{1-\left(t-x^{*}\right) h(t)}\right)
$$

must hold. In the latter case, for all $x \in I$,

$$
f(x)=c^{+}\left(x-x^{*}\right)^{\jmath} \exp \left(-j \int_{x}^{x^{+}} \frac{h(t) d t}{1-\left(t-x^{*}\right) h(t)}\right),
$$

which shows $f \in C^{k}(I)$. Since the two-parameter family defined in (3.2) contains all solutions of $N_{f}=T$ on $x<x^{*}$ and $x>x^{*}$ separately, the solution of $N_{f}=T$ is unique up to multiplication by a nonzero constant if $\delta \in\left\{0,1 / 2,1 / 3, \ldots, 1-k^{-1}\right\} \backslash\{1\}$.

If, on the other hand, $\delta>1-k^{-1}$, and correspondingly $\gamma>k$, then $f \in C^{k}(I)$ for any choice of the constants $c^{+}, c^{-}$, and $f\left(x^{*}\right)=f^{\prime}\left(x^{*}\right)=\cdots=f^{(k)}\left(x^{*}\right)=0$.

Case 3. Assume that $T^{\prime}(\operatorname{Fix}[T])=\{1\}$. If $\operatorname{Fix}[T]=I$, then trivially $T$ is the Newton map associated with $f \equiv 1$. Without loss of generality, therefore, assume that $x^{*}$ is the right boundary point of Fix [T]. In this case

$$
T(x)=x-\left(x-x^{*}\right)^{2} h(x),
$$

where $h \in C^{\infty}(I)$ and $h(x)>0$ whenever $x>x^{*}$, and $h(x)=0$ for all $x \in$ Fix [T]; in particular, therefore, $h\left(x^{*}\right)=0$. As before, pick $x^{+} \in I$ with $x^{+}>x^{*}$ and, analogously to (3.2), let

$$
f^{+}(x):= \begin{cases}\exp \left(-\int_{x}^{x^{+}} \frac{d t}{g(t)}\right) & \text { if } x>x^{*} \\ 0 & \text { if } x \leq x^{*}\end{cases}
$$

Using (3.3), with $\gamma$ replaced by $j$, and recalling that $g(t)=\left(t-x^{*}\right)^{2} h(t)$, it follows that $\lim _{x \backslash x^{*}}\left(x-x^{*}\right)^{-j} f^{+}(x)=0$ for all $j \in \mathbb{N}$. Thus $f^{+} \in C^{\infty}(I)$ and $N_{f^{+}}(x)=T(x)$ 

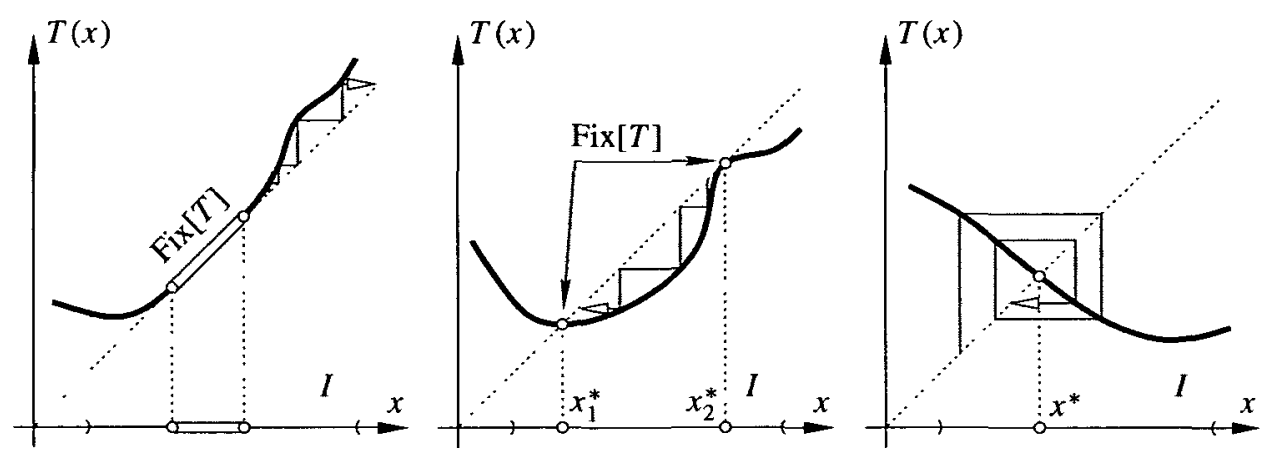

FIGURE 2. Three $C^{\infty}$ maps $T$ which are not Newton maps associated with any $C^{k}$ function on the interval $I$ because Fix $[T]$ is not attracting (left), Fix $[T]$ is not an interval (middle), and $T^{\prime}\left(x^{*}\right) \notin \Delta_{k}$ for any $k \in \mathbb{N}_{\infty}$, respectively.

whenever $x>x^{*}$ or $x \in \operatorname{Fix}[T]$. If Fix [T] has a left boundary point in $I$ as well, then define $f^{-}$in a "mirrored" manner and let $f=c^{+} f^{+}+c^{-} f^{-}$with nonzero constants $c^{+}, c^{-}$. Clearly, $f \in C^{\infty}(I)$ and $N_{f}=T$ for any choice of $c^{+}, c^{-}$.

The assertion concerning uniqueness up to multiplication by a constant is now obvious from the three cases detailed above.

COROLlary 3.3. Suppose $T \in C^{\infty}(I)$. Then $T$ is a Newton map, associated with $f \in C^{\infty}(I)$, if and only if $\operatorname{Fix}[T]$ is either empty or an attracting (possibly one-point) interval, and

$$
T^{\prime}(\operatorname{Fix}[T])=\left\{1-j^{-1}\right\}, \quad \text { for some } j \in \mathbb{N}_{\infty} \text {. }
$$

Moreover, $f$ is uniquely determined up to a multiplicative constant unless $j=\infty$ in (3.4) and the set $I \backslash$ Fix $[T]$ is not connected.

The next corollary requires $T$ to be not only $C^{\infty}$ but even real-analytic. Recall that a map is real-analytic if it can be represented by its Taylor's series in a neighbourhood of every point in its domain. Real-analytic Newton maps are especially easy to characterise. Although analyticity is a strong assumption indeed, the class of realanalytic functions is of great historical $[5,9]$ and practical relevance, as it contains, for example, all rational and trigonometric functions and compositions thereof $[1,4]$. If $f$ is real-analytic then so is $N_{f}$, provided the latter map is continuous $[1,2]$.

COROLLARY 3.4. Let $T$ be real-analytic on $I$, and $T(x) \not \equiv x$. Then $T$ is a Newton map, associated with a real-analytic function $f$, if and only if $T$ has at most one fixed point in $I$, and, in case a fixed point $x^{*}$ exists, $T^{\prime}\left(x^{*}\right)=1-j^{-1}$ for some $j \in \mathbb{N}$. Moreover, $f$ is unique up to multiplication by a constant. 
EXAMPLE 3.5. For $f(x)=\exp (-x)$ and $f_{j}(x)=x^{j}, j \in \mathbb{N}$, clearly $N_{f}(x)=x+1$ and $N_{f_{j}}(x)=\left(1-j^{-1}\right) x$, respectively. Thus all cases referred to in Corollary 3.4 can actually occur.

EXAMPLE 3.6. The much-studied logistic map $F_{\mu}(x)=\mu x(1-x)$ is a Newton map associated with a real-analytic function on $I=] 0,1$ [ if and only if $\mu \in M$, with $M:=]-\infty, 1] \cup\left\{1+j^{-1}: j \in \mathbb{N}\right\}$. Indeed, $F_{\mu}=N_{f_{\mu}}$ with functions

$$
f_{\mu}(x)=\left(\frac{x}{\mu x+1-\mu}\right)^{(1-\mu)^{-1}} \text { for } \mu \neq 1,
$$

and $f_{1}(x)=\exp \left(-x^{-1}\right)$. Note that while $f_{\mu}$ is real-analytic on $I$ for all $\mu \in M$, it is only in the trivial case $\mu=0$ that $f_{\mu}$ could be extended to a real-analytic function such that $N_{f_{\mu}}(x)=F_{\mu}(x)$ for all $x \in \mathbb{R}$. Consequently, $F_{\mu}$ is not a Newton map on $\mathbb{R}$ unless $\mu=0$.

EXAMPLE 3.7. It must be emphasised that Theorem 3.2 and Corollaries 3.3 and 3.4 do not force the set Fix [T] of a $C^{\infty}$ or real-analytic Newton map $T$ to attract all points in $I$. In fact, the map $T$ may at the same time exhibit some stable dynamical feature other than a fixed point. For a simple concrete example consider the (real-analytic) function

$$
f(x)=x \frac{3+x^{2}}{1+x^{2}}
$$

for which the associated Newton map

$$
N_{f}(x)=-\frac{4 x^{3}}{3+x^{4}}
$$

has the stable (in fact, super-attracting) 2-periodic orbit $\{\sqrt{3},-\sqrt{3}\}$.

REMARK. It is well known that if $f$ is a rational function (that is, a quotient of two polynomials) then $N_{f}$ can be extended uniquely to (and studied appropriately as) a smooth function $\bar{N}_{f}$ on $\overline{\mathbb{R}}$, the one-point compactification of $\mathbb{R}$. Though finite, $\operatorname{Fix}\left[\bar{N}_{f}\right]$ generally contains more than one point $[2,3]$. Corollary 3.4 , however, clearly still applies to $\operatorname{Fix}\left[\bar{N}_{f}\right] \cap I$ for every interval $I$ on which $f$ is real-analytic.

The above results about Newton maps have an immediate bearing on the distribution of the floating-point fractions of the iterates $x_{n}=N_{f}^{n}\left(x_{0}\right)$, that is, on the numerical data generated by NM. (See [7] for an account on the relevance of fraction parts distributions for practical computations.) In particular, this distribution depends significantly on the analytic properties of $N_{f}$ discussed in this note; the interested reader is referred to [4] for details. 


\section{Acknowledgements}

The first author was partially supported by a HuMBOLDT research fellowship. He wishes to thank the Johann Wolfgang von Goethe-Universität Frankfurt for its hospitality. The second author was partially supported by a National Security Agency Grant. He is grateful to the University of Leiden and the Universität Bonn, for invitations to visit in April 2005, during which time some of this research was carried out, and to the California Polytechnic State University, for its hospitality and its support as Research Scholar in Residence 2005-2006.

\section{References}

[1] S. Axler, P. Bourdon and W. Ramey, Harmonic Function Theory (Springer, New York, 1992).

[2] A. Beardon, Iteration of Rational Functions (Springer, New York, 1991).

[3] A. Berger, Chaos and Chance (deGruyter, Berlin New York, 2001).

[4] A. Berger and T. Hill, "Newton's method obeys Benford's law", Amer. Math. Monthly, to appear.

[5] P. Colwell, Solving Kepler's Equation Over Three Centuries (Willmann-Bell, Richmond, 1993).

[6] A. Katok and B. Hasselblatt, Introduction to the modern theory of dynamical systems (CUP, Cambridge, 1995).

[7] D. Knuth, The Art of Computer Programming, Vol. 2, 3rd ed. (Addison-Wesley, Reading, MA, 1997).

[8] W. Rheinboldt, Methods for Solving Systems of Nonlinear Equations, 2nd ed. (SIAM, Arrowsmith, Bristol, 1998).

[9] S. Smale, "Newton's method estimates from data at one point", in The Merging of Disciplines: New Directions in Pure, Applied and Computational Mathematics, (Springer, New York, 1986) 185-196. 